\title{
O sofrimento da população idosa com a insuficiência cardíaca: uma revisão narrativa
}

\author{
The suffering of the elderly population with heart failure: a narrative review
}

El sufrimiento de la población anciana con insuficiencia cardíaca: una revisión narrativa

Lucas Fernandes Soares Matos ${ }^{1 *}$, Davi Ramos do Nascimento ${ }^{1}$, Rege Farias Oliveira ${ }^{1}$, Eduardo Gama de Barros ${ }^{2}$, Marco Tulio Socola Juarez Oliveira ${ }^{3}$, Patrícia Oliveira Furbino ${ }^{4}$, Cecilia Lucia Carubia ${ }^{5}$, Angélica Gusmão Rocha ${ }^{6}$, Virgínia Soeiro Santos ${ }^{6}$, Andressa Dias de Andrade Fontes Amorim¹.

\section{RESUMO}

Objetivo: Compreender o impacto causado pela Insuficiência Cardíaca (IC) na população idosa e como a equipe multidisciplinar da atenção básica tem o papel no acompanhamento dessa população. Revisão bibliográfica: A IC é uma das principais patologias que acompanham o envelhecimento, gerando um importante impacto na qualidade de vida dos pacientes acometidos. Com o decorrer da doença, o indivíduo está propenso a ter dificuldade na realização das atividades instrumentais de vida diária. Além da doença física, a angústia, o medo e a dificuldade de lidar com as novas adaptações impostas pela doença, faz com que os idosos tenham um alto risco de desenvolver problemas psiquiátricos. As principais ações que são compartilhadas entre a equipe e o doente correspondem à manutenção do programa de exercícios físicos, contribuindo para manutenção da força muscular, flexibilidade e condicionamento cardiorrespiratório, além de ações educacionais para mudanças e adaptações no estilo de vida. Considerações finais: Dessa forma, este trabalho propôs abordar as mudanças físicas e emocionais que o idoso com IC vive, além do papel da equipe multidisciplinar no acompanhamento dessa população.

Palavras-chave: População idosa, Insuficiência cardíaca, Equipe multidisciplinar.

\begin{abstract}
Objective: To understand the impact caused by Heart Failure (HF) in the elderly population and how the multidisciplinary team of primary care plays a role in monitoring this population. Bibliographic review: HF is one of the main pathologies that accompany aging, generating an important impact on the quality of life of affected patients. As the disease progresses, the individual is likely to have difficulty performing instrumental activities of daily living. In addition to the physical illness, the anguish, fear and difficulty in dealing with the new adaptations imposed by the disease, make the elderly have a high risk of developing psychiatric problems. The main actions that are shared between the team and the patient correspond to the maintenance of the physical exercise program, contributing to the maintenance of muscle strength, flexibility and cardiorespiratory conditioning, in addition to educational actions for changes and adaptations in lifestyle. Final considerations: Thus, this work proposed to address the physical and emotional changes that elderly people with HF experience, in addition to the role of the multidisciplinary team in monitoring this population.
\end{abstract}

Keywords: Elderly population, Heart failure, Multidisciplinary team.

\footnotetext{
${ }^{1}$ Faculdade Santo Agostinho (FASA), Vitória da Conquista - BA. *E-mail: lucas-fsm@hotmail.com

2 Universidad Maimónides (UMAI), Caba - Buenos Aires, Argentina.

3 Universidad de Aquino Bolívia (UDABOL), Santa Cruz de La Sierra - Santa Cruz, Bolívia.

4 Universidad de Moron (UM), Morón - Buenos Aires, Argentina.

5 Universidad Nacional de Rosário (UNR), Rosário - Santa Fé, Argentina.

${ }^{6}$ Faculdade Pitágoras De Medicina De Eunápolis (FPME), Eunápolis - BA.
} 


\section{RESUMEN}

Objetivo: Comprender el impacto que produce la Insuficiencia Cardíaca (IC) en la población anciana y cómo el equipo multidisciplinario de atención primaria juega un papel en el seguimiento de esta población. Revisión bibliográfica: la IC es una de las principales patologías que acompañan al envejecimiento, generando un importante impacto en la calidad de vida de los pacientes afectados. A medida que avanza la enfermedad, es probable que el individuo tenga dificultades para realizar actividades instrumentales de la vida diaria. Además de la enfermedad física, la angustia, el miedo y la dificultad para afrontar las nuevas adaptaciones que impone la enfermedad, hacen que los ancianos tengan un alto riesgo de desarrollar problemas psiquiátricos. Las principales acciones que se comparten entre el equipo y el paciente corresponden al mantenimiento del programa de ejercicio físico, contribuyendo al mantenimiento de la fuerza muscular, flexibilidad y acondicionamiento cardiorrespiratorio, además de acciones educativas para cambios y adaptaciones en el estilo de vida. Consideraciones finales: Así, este trabajo se propuso abordar los cambios físicos y emocionales que experimentan las personas mayores con IC, además del rol del equipo multidisciplinario en el seguimiento de esta población.

Palabras clave: Población de edad avanzada, Insuficiencia cardíaca, Equipo multidisciplinario.

\section{INTRODUÇÃO}

O Brasil tem envelhecido de forma rápida e intensa, além disso, a maioria dos idosos tem se apresentado com baixo nível socioeconômico e com alta prevalência de doenças crônicas não transmissíveis. O processo de senescência, por si só, é responsável por alterações estruturais e funcionais de todos os sítios orgânicos, sendo o sistema cardiovascular um dos mais afetados, levando ao aumento da morbimortalidade e piora significativa da qualidade de vida dessa população (JÚNIOR LM, 2016).

As patologias cardiocirculatórias são fatores de predição para o declínio funcional nos idosos, o que pode antecipar e/ou exacerbar quadros demenciais. Isso se deve à influência de outros aspectos atrelados à idade, como: os sociais, econômicos e ambientais (VIANA PAS, et al., 2018). Dentre as doenças que afetam o aparelho circulatório, a Insuficiência Cardíaca (IC) é uma condição cada vez mais importante entre os idosos. A IC é a principal causa de internação em pessoas com mais de 65 anos, sendo 1,4 vezes mais comuns do que o Infarto Agudo do Miocárdio (IAM), e a segunda mais frequente nessa faixa etária (LUCCHESI P, et al., 2015).

A IC é uma síndrome complexa e heterogênea que causa alguma disfunção no coração. Essa ineficiência do coração de bombear sangue a um debito cardíaco que atenda a demanda metabólica dos tecidos, ou desempenha essa função a dispêndio de pressões ou volumes diastólicos elevados para conseguir manter as necessidades metabólicas do organismo. Os principais fatores de risco para a IC são um estilo de vida baseado em uma dieta alimentar rica em calorias, sal e gordura, tabagismo, sedentarismo, estresse, síndromes metabólicas como obesidade, dislipidemia, hipertensão arterial resistência à insulina e diabetes (ROHDE LE, et al., 2018)

A consequência é o desenvolvimento das etiologias da IC que pode ser divididas em três grupos, 0 primeiro, é composto pela fração de ejeção preservada (> 40 a 50\%), apresentando como doenças: hipertrofia patológica primaria (miocardiopatias hipertróficas) e secundaria (hipertensão arterial); envelhecimento; miocardiopatia restritiva, doenças infiltrativas (amiloidose, sarcoidose), doença do armazenamento (hemocromatose); fibrose e distúrbios endomiocárdicos (FERREIRA AG, et al., 2013).

O segundo grupo, é o da fração de ejeção reduzida (<40\%) compondo doenças como: Doença Arterial Coronariana (DAC), infarto agudo do miocárdio e isquemia miocárdica; sobrecarga pressórica crônica, hipertensão arterial e doença obstrutiva valvar; sobrecarga volumétrica crônica, doença valvar regurgitante, shunt intracardíaco, shunt extracardíaco; doença pulmonar crônica, cor pulmonale, alterações pulmonares vasculares; miocardiopatia dilatada não isquêmica, doenças familiares/genéticas, doenças infiltrativas; lesão induzida por toxinas/fármacos, doenças metabólica e viral; distúrbios da frequência e do ritmo, bradiarritmias e taquiarritmias crônicas. Por último tem se o estado de alto debito, como, doenças metabólicas, distúrbios nutricionais, exigência de fluxo sanguíneo excessivo e anemia crônica (SILVA WT, et al., 2020). 
E os sinais e sintomas clínicos comuns da IC são: dispneia, estertores, pressão venosa jugular elevada e edema e/ou manifestações de perfusão tissular inadequada como, por exemplo, intolerância aos esforços, fadiga, sinais de hipoperfusão e disfunção renal. Esse quadro é ocasionado pela capacidade funcional reduzida, responsável pela diminuição da qualidade de vida e do aumento da morbimortalidade da população mundial. Como é uma doença que demora muitos anos para apresentar algum tipo de sinal e sintoma, por se a via final de muitas doenças cardiovasculares, ela pode ser considera assim uma doença de população idosa (XAVIER SO e FERRETTI-REBUSTINI REL, 2019).

Assim, esse trabalho teve como objetivo compreender o impacto causado pela IC na população idosa e como a equipe multidisciplinar da atenção básica tem o papel no acompanhamento dessa população.

\section{REVISÃO BIBLIOGRÁFICA}

\section{Envelhecimento populacional}

Sabe-se que a pirâmide etária brasileira tem passado por uma intensa transformação nos últimos tempos. Fatores como a revolução técnico-científica influenciaram diretamente no aprimoramento da medicina, incluindo novidades nos métodos diagnósticos e de tratamento, o que reflete em um aumento da qualidade $e$ expectativa de vida da população em geral (VERAS RP e OLIVERIA M, 2018).

Além disso, a velhice não é uma doença, e sim uma etapa em que ocorre modificações celulares importantes no indivíduo, conhecido como processo de senescência. Várias são as mudanças ocorridas na velhice, tanto no âmbito orgânico, envolvendo alterações metabólicas e funcionais, como no ambiente social, o que faz com que o idoso passe por um processo de readaptação à nova realidade inserida (TEIXEIRA SMO, et al., 2015; ANDRADE CCF, et al., 2020).

Características como diminuição do nível de hidratação e elasticidade da pele fazem com que o indivíduo manifeste uma aparência específica para a idade; há uma maior dificuldade para locomoção, o que pode ser atribuído à atrofia muscular e esclerose das articulações. Ocorre também uma maior perda da mineralização óssea, predispondo a ocorrência de fraturas à pequenos impactos. Além do mais, o idoso é vulnerável ao desenvolvimento de doenças degenerativas, como as que envolvem o aparelho cardiovascular (angina, insuficiência cardíaca, infarto agudo do miocárdio); cerebrovasculares (acidente vascular encefálico); neurovegetativas (demência, doença de Parkinson); câncer e transtornos mentais como depressão (GOMES DTB, et al., 2020; LIMA-COSTA MF, 2018).

É válido destacar que há uma contribuição sociocultural para o estabelecimento dessas condições clínicas. Com o passar dos anos, ocorre uma reestruturação do ambiente social do indivíduo, sendo que alguns possuem uma menor capacidade de adaptação à essa nova realidade, fatores diversos estão envolvidos, como: aposentadoria, maior tempo livre, síndrome do ninho vazio, morte do companheiro(a), julgamento social e perda da segurança econômica. É partindo deste pressuposto que as ações de saúde pública se tornam essenciais, com o objetivo de promover um acompanhamento necessário para a garantia da qualidade de vida dessa população (PAZ JMB, 2021).

Os idosos necessitam de uma abordagem diferente daquelas dos adultos mais jovem, algo que ainda é um desafio na contemporaneidade. Um dos principais problemas vigentes é a fragmentação desnecessária da atenção aos idosos, com múltiplas consultas de especialistas, exames clínicos e de imagem, polifarmácia e outros procedimentos, contrariando a ideia de prevenção quaternária, o que contribui para uma sobrecarga do sistema de saúde. Isso pode acarretar em um grande impacto financeiro e organizacional e não gera benefícios significativos à qualidade de vida (VERAS RP e OLIVERIA M, 2018).

A maioria dos modelos assistenciais à saúde ainda é o tradicional, focando majoritariamente na moléstia e na cura, negligenciando uma série de vulnerabilidades que contribuem para o declínio orgânico e mental da população idosa. Assim, faz-se necessário que o acompanhamento médico seja oferecido de maneira integral e compartilhada, valorizando a autonomia do indivíduo doente, ou seja, o modelo de atenção mais adequado para a população idosa é aquele que apresenta uma proposta de linha de cuidado, envolvendo todos os aspectos biopsicossociais (VERAS RP, et al., 2013). 


\section{Insuficiência cardíaca}

A IC é uma das doenças mais prevalentes em todo o mundo, cuja incidência aumenta com o envelhecimento populacional e propagação dos fatores de risco cardiovasculares. Possui uma grande morbidade quando não tratada, podendo limitar o indivíduo na realização das Atividades Básicas de Vida Diária (ABVD) e predispondo à eventos malignos, com o aumento dos índices de mortalidade (FREITAS AKE e CIRINO RH, 2017).

Ademais, a IC pode ser classificada como de baixo débito (mais prevalente), que seria quando o coração tem um defeito estrutural ou funcional que faz com que haja dificuldade de bombeamento, ou de alto débito, em que alguma condição sistêmica faz com que os tecidos necessitem de uma quantidade maior de oxigênio, exigindo uma atuação cardíaca mais vigorosa, situações clínicas como anemia, tireotoxicose e fístulas arteriovenosas podem estar associadas (SOUSA MM, et al., 2017).

Critérios da American Heart Association (AHA) classificam a IC em quatro estágios: estágio A inclui os pacientes que possuem alto risco de desenvolver IC, mas ainda sem a doença estrutural ou sintomas de IC. $O$ estágio $B$ inclui os pacientes que já possuem uma alteração cardíaca, mas permanecem assintomáticos, como aqueles com IAM prévio. O estágio $C$ inclui o paciente que apresenta alteração cardíaca estrutural concomitante com sintomas de IC. Por fim, o estágio D corresponde aos pacientes refratários ao tratamento e que necessitam de intervenção especial, como um transplante cardíaco (FERREIRA AG, et al., 2013).

Outra forma de classificação se baseia na tolerância ao exercício, no paciente que possui o diagnóstico de IC. A New York Heart Association (NYHA) classifica os pacientes em Classes Funcionais (CF): A CF I corresponde à ausência de sintomas. A CF II são sintomas desencadeados por atividades cotidianas. A CF III são sintomas causados por atividades menos intensas do que as cotidianas ou aos pequenos esforços. A CF IV corresponde à sintomas presentes aos mínimos esforços ou no repouso (ROHDE LE, et al., 2018).

É de fundamental importância a realização de um exame clínico minucioso, levando em consideração os principais fatores de risco, de agravamento e características clínicas, que podem predizer uma etiologia, além de ofertar dados relevantes para o manejo apropriado (LUCCHESI P, et al., 2015).

Os sinais e sintomas da IC vão variar a depender da sua fisiopatologia. Se houver um comprometimento da ejeção do ventrículo esquerdo (IC esquerda), os sintomas serão de congestão pulmonar, podendo se manifestar com dispneia aos esforços, tosse, taquipneia, ortopneia, dispneia paroxística noturna e sinais como estertores pulmonares e terceira bulha. Ademais, se o defeito for no ventrículo direito (IC direita) as manifestações serão de congestão sistêmica, podendo ocorrer edema periférico, elevação da pressão venosa jugular, refluxo hepatojugular, hepatomegalia dolorosa, derrame pleural e ascite (MARTÍNEZ-BRANA L, et al., 2015).

Em relação aos marcadores da IC, destacam-se os peptídeos natriuréticos BNP e NT-proBNP, os quais possuem relevância para o diagnóstico, apesar que seus números podem estar alterados em outras condições clínicas, como insuficiência renal crônica e anemia, podendo levar à falso-positivos. Os níveis dos peptídeos natriuréticos tendem a aumentar de forma progressiva com a piora da classe funcional do paciente, sendo mais elevados na IC com Fração de Ejeção Reduzida (ICFER) do que na IC com Fração de Ejeção Preservada (ICFEP). O ponto de corte mais aceitável para o diagnóstico é o BNP acima de $35 \mathrm{pg} / \mathrm{ml}$, acarretando em uma maior sensibilidade e reduzindo falso-negativos (ROHDE LE, et al., 2018).

O exame de imagem de escolha para o diagnóstico e acompanhamento do paciente é o ecocardiograma transtorácico. O mesmo permite uma avaliação precisa da função ventricular sistólica e diastólica, além de mensurar a espessura miocárdica, o tamanho das cavidades, a conformação e função das válvulas e doenças do pericárdio. Outros exames como o eletrocardiograma e a radiografia de tórax podem ser úteis para avaliar o ritmo cardíaco e identificar um possível IAM, e na visualização de uma possível cardiomegalia e congestão pulmonar, respectivamente (MONTERA WM, et al., 2012; NASCIMENTO WO, et al., 2016).

Os principais objetivos no tratamento da IC é a reabilitação da condição clínica do paciente e da capacidade funcional, além da garantia da qualidade de vida, impedindo a progressão da doença e reduzindo 
as taxas de mortalidade. O acompanhamento integral do paciente com IC é de fundamental importância para o desfecho clínico, principalmente aqueles feitos por uma equipe multiprofissional (FREITAS AKE e CIRINO $\mathrm{RH}, 2017)$.

A terapia da IC é dividida em duas vertentes que se completam: tratamento não farmacológico e o tratamento farmacológico. Lembrando que, identificar e corrigir a causa da IC é essencial, pois muitas etiologias são tratáveis e reversíveis, tendo uma importância significativa para o prognóstico do paciente (ALBUQUERQUE DC, et al., 2015). Dentre as medidas não farmacológicas, algumas orientações são imprescindíveis, como: dieta com baixo teor de sódio, restrição hídrica (ingestão de aproximadamente 1 a 1,5 litros de água/dia), além do monitoramento do peso corporal. O consumo de álcool moderado é permitido, exceto em pacientes com miocardiopatia alcóolica. Já em relação ao tabaco, o uso deve ser interrompido, tanto da forma ativa quanto da passiva (SOUSA MM, et al., 2021).

A vacinação anual contra influenza e pneumococo é recomendada para todos os pacientes com IC. É comprovado que essa medida reduz o número de internações por doenças cardiovasculares. Em relação à prática de atividades físicas, exercícios leves são recomendados, proporcionando o aumento da capacidade funcional do paciente (ROHDE LE, et al., 2018; MENDES LP, et al., 2016).

Várias medicações podem ser utilizadas na IC, algumas com o intuito de aliviar o quadro agudo, sem diminuição de mortalidade, e outras com benefícios à longo prazo, as quais atuam diretamente no recondicionamento da fração de ejeção e no remodelamento cardíaco (MONTERA WM, et al., 2012).

Os inibidores da Enzima Conversora de Angiotensina (IECA) devem ser prescritos a todos os pacientes, exceto em casos de contraindicação, sendo que as doses devem ser administradas em pequenas quantidades inicialmente e aumentadas de forma progressiva. Em casos de efeitos adversos, como tosse ou angioedema, a utilização de um bloqueador do receptor de angiotensina (BRA) é indicado. Além disso, os betabloqueadores (BB) são excelentes alternativas em pacientes que possuem doença arterial coronariana associada, devido à redução do antagonismo da atividade simpática, porém é contraindicado em casos de asma grave e bradicardias. Os medicamentos antagonistas da aldosterona podem ser utilizados em pacientes sintomáticos com disfunção sistólica, diminuindo a pré-carga, porém não costumam ser prescritos para aqueles que já fazem uso de iECA ou BRA, pelo risco de hipocalemia (SOUSA MM, et al., 2021).

Para aliviar os principais sintomas da IC, como o edema periférico, medicamentos que promovem a natriurese podem ser indicados, promovendo a melhora do estado volêmico. Em pacientes com um quadro de edema mais intenso, diuréticos de alça como a furosemida são geralmente utilizados, possuindo efeito imediato. Em quadros mais leves, pode-se fazer o uso de diuréticos tiazídicos como, exemplo, a hidroclorotiazida (ALBUQUERQUE DC, et al., 2015).

A digoxina, da classe dos digitálicos, são medicamentos utilizados em casos de refratariedade ao tratamento à longo prazo, possuindo um papel arritmogênico e uma ação anticolinérgica. Porém, essa classe de medicamentos causa extensos efeitos adversos, como náuseas, vômitos, alterações visuais e arritmias. Ademais, as drogas vasoativas (noradrenalina) são utilizadas em pacientes muito graves com risco de vida, apresenta um potente efeito vasoconstritor. Pode ser utilizado em casos de choque cardiogênico (ROHDE LE, et al., 2018; SILVA LA, et al., 2010).

Pacientes no estágio $D$ da $A H A$, que já não respondem ao tratamento medicamentoso, podem ser beneficiados pelo uso de alguns dispositivos, como: ressincronizador biventricular, cardiodesfibriladores implantáveis e dispositivos de assistência ventricular. Se não houver mais alternativas, o transplante cardíaco pode ser pensado (ALBUQUERQUE DC, et al., 2015; TAVARES RE, et al., 2017).

\section{Repercussões da IC no paciente idoso e sua relação com doença demencial}

Com o aumento exponencial da expectativa de vida, doenças crônicas, sobretudo cardiovasculares, têm ganhado destaque. A IC é uma das principais patologias que acompanham o envelhecimento, gerando um importante impacto na qualidade de vida dos pacientes acometidos, assim como $o$ alto índice de internações, sobrecarregando o sistema de saúde (DOURADO MB, et al., 2019). 
O envelhecimento está associado às modificações anatomo-fisiológicas que determinam diminuição da capacidade funcional sistêmica e a capacidade de manter um equilíbrio homeostático frente às situações adversas. Assim, deixando o indivíduo vulnerável em termos de saúde física e mental, aumentando os índices de dependência física e isolamento social (VENTURA CF, 2020; MESQUITA ET, et al., 2017).

Em geral, pessoas com IC sofrem uma mudança importante no seu padrão de vida prévio. Os sinais e sintomas da doença, como dispneia, ortopneia, dor, desconforto precordial, síncope, taquicardia, fadiga e edema são os principais fatores que limitam o desempenho funcional. Com o decorrer da doença, o indivíduo está propenso a ter dificuldade na realização das atividades instrumentais de vida diária, como manter as tarefas domésticas, preparar refeições, fazer compras, gerenciar finanças, dirigir e usar o telefone e outros aparelhos de comunicação. Portanto, a associação da IC com o declínio funcional fisiológico, gera uma sobreposição de fatores que contribuem à um declínio progressivo e sistêmico da qualidade de vida (NETO JMR, et al., 2020; SILVA MMBS, et al., 2021).

Além da doença física, a angústia, o medo e a dificuldade de lidar com as novas adaptações impostas pela doença, faz com que os idosos tenham um alto risco de desenvolver problemas psiquiátricos, como depressão e ansiedade. A prevalência de depressão entre os pacientes com IC varia entre $10 \%$ a $40 \%$, associada à um importante aumento da mortalidade e declínio funcional (RODRIGUES GHP, et al., 2015).

Pacientes com diagnóstico estabelecido de IC, apresentam uma diminuição do fluxo sanguíneo cerebral por conta da limitação do bombeamento cardíaco, o que pode acelerar e/ou precipitar o desenvolvimento de déficits cognitivos. Cerca de $75 \%$ da população com IC apresenta disfunção cerebral e comprometimento cognitivo. Correlacionando-se a função cognitiva com a gravidade da IC quantificada pela NYHA, pacientes nas classes funcionais II e III da NYHA podem apresentar comprometimento cognitivo moderado-grave e até $50 \%$ dos doentes com classe funcional IV apresentam déficit cognitivo extremamente avançado (XAVIER SO, et al., 2015).

A IC afeta de forma negativa vários domínios cognitivos, os principais são a atenção, capacidade de aprendizagem, memória de trabalho e recordação, velocidade psicomotora e função executiva. Na prática, esses pacientes apresentam uma memória recente prejudicada, além de uma menor capacidade de realização de atividades básicas de vida, como tomar banho, se alimentar, se locomover e manter 0 autocuidado, o que gera uma diminuição importante da autonomia (SANTOS CS, et al., 2020).

A descompensação da IC é um reflexo direto dessa conjuntura, devido, principalmente, à uma má adesão terapêutica, podendo estar associado ao esquecimento, dificuldade na interpretação e manuseio de medicamentos e hábitos de dieta inadequados, viés que pode ser complementado por outros fatores, como baixo nível socioeconômico e baixa escolaridade. Repetidas internações fazem com que os idosos fiquem mais vulneráveis e dependentes, diminuam seu contato diário com o meio social e, como consequência, estabelece-se uma diminuição progressiva da sua capacidade neurocognitiva e funcional, em consonância com a debilidade cardíaca progressiva, tornando um ciclo vicioso e aumentando a morbimortalidade dos doentes (BARBOSA RR, et al., 2018; ULBRICH AZ, et al., 2013).

\section{Papel da equipe multidisciplinar no acompanhamento do paciente idosos com IC}

Atualmente, a assistência ao idoso apresente diversas fragilidades no processo organizacional e operacional, começando pela fragmentação do sistema, em que a doença crônica específica é vista como o centro do cuidado, em detrimento da autonomia e particularidades de cada paciente. Para que se tenha garantia de um acompanhamento eficaz é necessária uma atenção voltada de maneira integral, onde a equipe multidisciplinar possa elaborar uma rede de cuidados que vai desde a entrada no sistema até o fim da vida do indivíduo (MEDEIROS KKAS, et al., 2017)

O modelo adequado de atenção aos idosos é aquele que valoriza ações educativas, promoção de saúde, postergação de moléstias e reabilitação precoce. A equipe multidisciplinar da atenção básica deve ajudar o paciente a identificar e entender suas principais necessidades e limitações, implementando ações que contribuam para o bem-estar físico e emocional do doente (VENTURA CF, 2020). 
As relações de cuidado se tratando de uma doença crônica envolvem circunstância especiais, que vai desde um contato interpessoal saudável, com o objetivo de desenvolver confiança, e a valorização das demandas do paciente e da sua família, para que sejam identificadas e atendidas com êxito e empatia. Essa é uma fase crítica para a implementação de um plano de cuidados de longa duração (MEDEIROS KKAS, et al., 2017).

No paciente com diagnóstico de IC, os seguintes itens devem ser priorizados: estratificação de risco préalta com o objetivo de identificar pacientes com maior chance de descompensação; manter a estabilidade do doente com a devida otimização do tratamento, iniciando e titulando medicamentos conforme a melhor resposta e adaptação; identificação e tratamento de etiologias de base; consideração para utilização de dispositivos cardíacos em casos excepcionais; envolvimento mútuo do paciente e dos seus familiares e cuidadores em campanhas educativas sobre a IC e programa de reabilitação cardíaca (VENTURA CF, 2020).

Assim, a reabilitação cardíaca é formada por um conjunto de atividades necessárias para melhora das condições físicas, mentais e sociais dos pacientes cardiopatas, contribuindo para o seu retorno gradual à comunidade e proporcionando uma maior qualidade de vida e autonomia. A reabilitação cardíaca tem o enfoque principal na mudança do estilo de vida, cabendo ao profissional de saúde as recomendações e o acompanhamento dos hábitos cotidianos do paciente (RÊGO MLM, et al., 2018).

As principais ações que são compartilhadas entre a equipe e o doente correspondem à manutenção do programa de exercícios físicos, contribuindo para manutenção da força muscular, flexibilidade e condicionamento cardiorrespiratório, além de ações educacionais para mudanças e adaptações no estilo de vida. Vale ressaltar que os pacientes com IC devem receber orientações sobre uma alimentação saudável, com baixa ingesta hidrossalina e lipídica para evitar descompensações (RÊGO MLM, et al., 2018).

\section{CONSIDERAÇÕES FINAIS}

Dessa forma, este trabalho propôs abordar a importância da relação entre a IC e o envelhecimento populacional, ressaltando-se a crescente mudança do perfil epidemiológico nos últimos anos e a associação com doenças crônicas, o que gera maiores gastos e saturação do serviço público de saúde. Além disso, como as mudanças ocorridas no aparelho cardiovascular com o envelhecimento podem ter repercussões físicas, emocionais e sociais nos idosos que convivem com a IC e como a equipe multidisciplinar da atenção básica tem o papel importante no acompanhamento e direcionamento dessa faixa etária, oferecendo, assim, a garantia da saúde e qualidade de vida.

\section{REFERÊNCIAS}

1. AGUIAR NM. Avaliação da qualidade de vida dos pacientes com insuficiência cardíaca. Revista Eletrônica Acervo Científico, 2021; 32: e8404.

2. ALBUQUERQUE DC, et al. I Registro Brasileiro de Insuficiência Cardíaca - Aspectos Clínicos, Qualidade Assistencial e Desfechos Hospitalares. Arquivos Brasileiros de Cardiologia; 2015; 104(6): 433-442.

3. ANDRADE CCF, et al. Treinamento Muscular Inspiratório Como Recurso Para a Proteção Do Indivíduo Com Insuficiência Cardíaca. Psicologia e Saúde em debate, 2020; 6(1): 49-59.

4. BARBOSA RR, et al. Insuficiência cardíaca na população geriátrica: dados de um registro unicêntrico. Revista da Sociedade Brasileira de Clínica Médica, 2018; 16(4): 203-7.

5. DOURADO MB, et al. Perfis clínico e epidemiológico de idosos com insuficiência cardíaca. Revista de Enfermagem UFPE Online, 2019; 13(1): 408-15.

6. FERREIRA AG, et al. A doença arterial coronariana e o envelhecimento populacional: como enfrentar esse desafio? Revista Hospital Universitário Pedro Ernesto, 2013; 12(1): 13-24.

7. FREITAS AKE, CIRINO RH. Manejo ambulatorial da insuficiência cardíaca crônica. Revista Médica da UFPR, 2017; 4(3): 123-136.

8. GOMES DTB, et al. Comorbidades entre idosos tabagistas. Editora Cientifica Digital, 2020; 1: 219-228.

9. JÚNIOR LM. O envelhecimento e o coração: as valvas. Revista da Faculdade de Ciências Médicas de Sorocaba, 2016; 18(1): 58-59. 
10. LIMA-COSTA MF. Envelhecimento e saúde pública: o Estudo Longitudinal do Envelhecimento Brasileiro (ELSI-Brasil). Revista de Saúde Pública. 2018; 52(2): 1-3.

11. LUCCHESI P, et al. Insuficiência cardíaca como preditor de dependência funcional em idosos hospitalizados. Rev. esc. Enferm., 2015; 49(5)

12. MARTÍNEZ-BRANA L, et al. Características clínicas e prognóstico da insuficiência cardíaca em pacientes idosos. Revista Portuguesa de Cardiologia; 2015; 34(7-8): 457-463.

13. MEDEIROS KKAS, et al. O desafio da integralidade no cuidado ao idoso, no âmbito da Atenção Primária à Saúde. Revista Saúde Debate, 2017; 41(3): 288-295.

14. MENDES LP, et al. Avaliação do estado nutricional e consumo alimentar em pacientes com Doença de Alzheimer. Revista da universidade vale do rio verde, 2016; 14(2): 502-515.

15. MESQUITA ET, et al. Entendendo a Hospitalização em Pacientes com Insuficiência Cardíaca. International Journal of Cardiovascular Sciences, 2017; 30(1): 81-90.

16. MONTERA WM, et al. Resumo da II Atualização das Diretrizes Brasileiras de Insuficiência Cardíaca Aguda $2009 / 2011$. Arquivos Brasileiros de Cardiologia, 2012; 98(5):375-383.

17. NASCIMENTO WO, et al. Perfil do idoso com insuficiência cardíaca internado em um hospital de urgência. Cogitare Enfermagem, 2016; 21(4): 01-10.

18. NETO JMR, et al. Insuficiência cardíaca aguda. Revista Sociedade de Cardiologia do Estado de São Paulo, 2020; 30(2): 147-57.

19. PAZ JMB. Tecnologia educacional sobre viver melhor com insuficiência cardíaca: estudo de validação. Revista Online de Pesquisa, 2021; 13: 428-433.

20. RÊGO MLM, et al. Déficit Cognitivo na Insuficiência Cardíaca e os Benefícios da Atividade Física Aeróbia, I. Arquivo Brasileiro de Cardiologia, 2018; 110(1): 91-94.

21. RODRIGUES GHP, et al. Depressão como determinante clínico de dependência e baixa qualidade de vida em idosos cardiopatas. Arquivos Brasileiros de Cardiologia, 2015; 104: 443-449.

22. ROHDE LE, et al. Diretriz Brasileira de Insuficiência Cardíaca Crônica e Aguda. Arquivos Brasileiros de Cardiologia, 2018; $111(3):$ 436-539.

23. SANTOS CS, et al. Fatores associados à demência em idosos. Revista Ciência e Saúde Coletiva, 2020; 25(2): 603611.

24. SILVA MMBS, et al. Qualidade de vida de idosos com insuficiência cardíaca. Revista Ciência y enfermería, 2021; 27(8): 1-11.

25. SILVA LA, et al. Perfil clínico de pacientes com insuficiência cardíaca inseridos em reabilitação precoce no serviço de urgência e emergência. Revista Sociedade de Cardiologia do Estado de São Paulo, 2010; 30(2): 279-279.

26. SILVA WT, et al. Características clínicas e comorbidades associadas à mortalidade por insuficiência cardíaca em um hospital de alta complexidade na Região Amazônica do Brasil. Revista Pan-Amazônica de Saúde, 2020; 11: e202000449.

27. SOUSA MM, et al. Comunicação persuasiva e redução do consumo de sal em pessoas com insuficiência cardíaca: estudo-piloto. Revista Brasileira de Enfermagem, 2021; 74(2): e20200715.

28. SOUSA MM, et al. Qualidade de vida de pacientes com insuficiência cardíaca: revisão integrativa. Revista de enfermagem UFPE online, 2017; 11(3):1289-98.

29. TAVARES RE, et al. Envelhecimento saudável na perspectiva de idosos: uma revisão integrativa. Revista brasileira de geriatria e gerontologia, 2017; 20(6): 889-900.

30. TEIXEIRA SMO, et al. Reflexões acerca do estigma do envelhecer na contemporaneidade. Estudos Interdisciplinares sobre o Envelhecimento, 2015; 20(2): 503-515.

31. ULBRICH AZ, et al. Capacidade funcional como preditor de qualidade de vida na insuficiência cardíaca. Fisioterapia em movimento, 2013; 26(4): 845-853.

32. VENTURA CF. Envelhecimento, qualidade de vida e bem-estar subjetivo: percepções de idosos participantes de um grupo social. New Trends in Qualitative Research, 2020; 3: 927-935.

33. VERAS RP, et al. Modelos de atenção à saúde do idoso: repensando o sentido da prevenção. Physis: Revista de Saúde Coletiva, 2013; 23(4): 1189-1213.

34. VERAS RP, OLIVERIA M. Envelhecer no Brasil: a construção de um modelo de cuidado. Ciência em saúde coletiva; 2018; 23(6): 1929-1936.

35. VIANA PAS, et al. Perfil de pacientes internados para tratamento de insuficiência cardíaca descompensada. SANARERevista de Políticas Públicas, 2018; 17(1): 15-23.

36. XAVIER SO, et al. Insuficiência cardíaca como preditor de dependência funcional em idosos hospitalizados. Revista da escola de Enfermagem USP, 2015; 49(5): 790-796.

37. XAVIER SO, FERRETTI-REBUSTINI REL. Características clínicas da Insuficiência Cardíaca associadas à dependência funcional admissional em idosos hospitalizados. Revista Latino-Americana de Enfermagem, 2019; 27: e3137. 